végétation et les groupements muscinaux des montagnes d' Algesiras. Mém. Mus. Nat. His. Nat. 21:85-116. Paris.

BUCH, H. -1942- Deux mousses critiques de la Péninsule Ibérique. Rev. Bryol. Lichénol. 13: 45-48. Paris.

CASARES, M. -1915- Enumeración y distribución geográfica de las Muscineas de la Península Ibérica. Trab. Mus. Nac. Cienc. Nat. Ser. Bot. 2: 1-179. Madrid.

CASAS, C. -1975- Brioteca Hispanica 1970. Acta Phytotax. Barc. 15:27-33. Barcelona.

CASAS,C., M. BRUGUES, R. CROS \& C. SERGIO -1992- Bryophyte Cartography. III: 101-150. Institut d'Estudis Catalans. Barcelona.

JOVET-AST \& H. BISCHLER - 1976- Hépatiques de la Péninsule Ibérique: Enumération, Notes écologiques. Rev. Bryol. Lichénol. 42: 931-987. Paris.

LUISIER, A. -1915- Fragments de Bryologie Ibérique. 8. Espèces nouvelles pour la flore espagnole. Broteria Ser. Bot. 13:154-157. Braga.

LUISIER, A. -1918- Fragments de Bryologie Ibérique. 14. Mousses de Galice. Brot. Ser. Bot. 16: 123-142. Braga.

REINOSO, J. - 1984- Contribución al conocimiento de la flora briofítica de Galicia. Briófitos de la fraga de Caaveiro, I. Musgos. Lazaroa 6: 237247. Madrid.
REINOSO, J. y J. ALVAREZ - 1984- Estudio de la flora briológica de la cuenca del río de Rojos (A Coruña). Acta Científica Compostelana 21:273284. Santiago de Compostela.

REINOSO, J. y M. SMITH - 1985- Estudio briológico de la ciudad de Santiago. Trabajos Compostelanos de Biología 12: 185-196. Santiago de Compostela.

REINOSO, J. -1998- Sematophyllum substrumulosum (Hampe) Britt. en España. Stvdia Botanica 17:131-132. Salamanca.

SERGIO, C., C. CASAS, M. BRUGUES \& R.M. CROS - 1994- Lista Vermelha dos Briofitos da Península Ibérica. Instituto da Conservaçao da Natureza. Museu, Laboratório e Jardim Botânico. Lisboa.

Aceptado para su publicación en mayo de 2000

Dirección de los autores. J. REINOSO FRANCO: Dpto. de Bioloxía Vegetal (Botánica). Facultade de Bioloxía. Universidade de Santiago de Compostela. 15706, Santiago de Compostela. A. GARCÏA MOLARES: Dpto. de Recursos Naturais e Medio Ambente. Facultade de Ciencias. Universidade de Vigo. 36200, Vigo. España.

\title{
88. NOTAS SOBRE ORQUÍDEAS DE ALICANTE
}

Manuel B. CRESPO, Joan PIERA y Michael R. LOWE

Notes on the Orchids of Alicante.

Palabras clave. Corología, flora, Orchidaceae, Alicante, España.

Key words. Chorology, flora, Orchidaceae, Alicante, Spain.

Como resultado de los estudios que se vienen realizando en los últimos años sobre las orquídeas de la provincia de Alicante, se aportan nuevas localidades de táxones críticos 
o interesantes. La revisión de referencias previas y el trabajo de campo realizado durante el último año permite, por un lado, ampliar la distribución alicantina de algunos táxones poco conocidos, y, por otro lado, presentar algunas novedades para el catálogo provincial.

El tratamiento taxonómico de las plantas citadas se ajusta al propuesto por Delforge (1994) y Mateo \& Crespo (1998). Los pliegos testigo del material citado están depositados en el herbario $\mathrm{ABH}$ de la Universidad de Alicante. En algunos casos se indica la existencia de material fotográfico que justifica las localidades indicadas.

\section{Cephalanthera damasonium (Mill.) Druce}

ALICANTE: Alcoy, Carrascal de la Font Roja, 30SYH1282, 1150 m, 10-VII-2000, M. R. Lowe, J. Piera \& M. B. Crespo (ABH 43655). Ibídem [30SYH18], 17-V-1964, A. Rigual (ut Epipactis latifolia, ABH 20123). Villena, Sierra de Salinas, pr. Cañada del Enebral, 30SXH7363, 950 m, 4-VII1993, A. Rigual, M. B. Crespo \& al. (ABH 6480). Ampliación de área.

Serra \& Mateo (1994) identificaron como $C$. damasonium un espécimen recolectado por $\mathrm{J}$. Mansanet y G. Mateo en la Font Roja de Alcoi, que se conserva, sin numerar, en el herbario VAL (antes VAB). Sin embargo, recientemente Serra et al. (2000) corrigen la identificación del citado material -que ahora atribuyen a C. rubra (L.) L.C. Rich.-, e indican la existencia de $C$. damasonium en sólo dos localidades alicantinas: $S^{\text {a }}$ del Rentonar y Puig Campana, donde fue indicada por Solanas (1998).

Por nuestra parte, ampliamos aquí considerablemente la distribución de $C$. damasonium hacia el extremo noroccidental de la provincia y confirmamos también su existencia en la Font Roja de Alcoi. En esta última localidad se ha detectado una notable población de unos 50 ejemplares, muchos de ellos con numerosos tallos. Habitan, junto con $C$. rubra, en una zona de antiguos campos de cultivo -actualmente colonizados por matorral serial-, circundados por un bosquete abierto de Quercus rotundifolia Lam. No obstante, es importante destacar que en el herbario $\mathrm{ABH}\left(\mathrm{n}^{\circ}\right.$ 20123) existen ejemplares de esta especie recolectados por A. Rigual en el mismo territorio, pero que fueron identificados como Epipactis latifolia (L.) Hall y, posteriormente, publicados como E. helleborine (L.) Crantz (cf. Rigual, 1972).

\section{Epipactis cardina Benito \& C.E. Hermos.}

ALICANTE: Alcoi, Sierra del Carrascal de la Font Roja, 30SYH1282, 1150 m, 10-VII-2000, M.R. Lowe, J. Piera \& M.B. Crespo (ABH 43657). Ibídem, [30SYH18], 17-VII-1957, A. Rigual (ABH 23586). Ibídem, pr. Menejador, 30SYH1482, $1280 \mathrm{~m}, 30$ VI-1997, J.L. Solanas \& al. (ABH 37883). El Algarejo, Sierra del Reclot, 30SXH7748, $985 \mathrm{~m}$, 10-VII-2000, M.R. Lowe, J. Piera \& M.B. Crespo (archivo fotográfico). Sierra del Maigmó, [30SYH06], 900 m, 26-IX-1957, A. Rigual (ABH 23589). Monóvar, Sierra del Reclot, 30SXH7848, $950 \mathrm{~m}, 30-\mathrm{V}-1997$, A. Navarro \& M.B. Crespo (ABH 34989). $2^{\text {a }}$ citas provinciales y ampliación de área.

Planta recientemente descrita de la provincia de Teruel (Benito Ayuso \& Hermosilla, 1998) e indicada de la Sierra del Carrascar, Alcoi (Serra et al., 2000). El estudio de los materiales de este género depositados en $\mathrm{ABH}$ ha revelado la existencia de varios especímenes pertenecientes a Epipactis cardina.. Igualmente, se ha confirmado la existencia de esta especie en dos localidades, por observación directa. Hasta la fecha no se han podido confirmar las menciones previas de E. helleborine (L.) Crantz en Alicante, que presumiblemente sean erróneas, como ya sugerían Mateo \& Crespo (1998). Sin embargo, algunos ejemplares robustos procedentes de la Font Roja, observados en el herbario y en el campo, requieren nuevos estudios, ya que presentan afinidades con E. tremolsii Pau.

\section{Ophrys arnoldii P. Delforge}

ALICANTE: Benimantell, Serra de l'Aixortà, Cerro de los Parados, 30SYH4687, 1025 m, 29-V2000, M. R. Lowe \& J. Piera (archivo fotográfico). Benissa, Serra de Bèrnia, pr. El Forat, 30SYH5783, $900 \mathrm{~m}, 1-\mathrm{V}-1991$, M. B. Crespo (ABH 0626). Ibídem, camí de Bèrnia al Forat, 30SYH5883, 640 m, 15IV-2000, J. Piera (ABH 43881). Ibídem, 30SYH5983, 700 m, 1-V-1996, M. R. Soler \& J. C. Cristóbal (ABH 33511). Ibídem, crestas de Bèrnia, 30SYH5982, 900 m, 21-IV-2000, M. R. Soler \& J. C. Cristóbal (ABH 32998). Benissa, Pinos (al norte de Bèrnia), 30SYH6085, 340 m, 29-IV-1994, J.Piera (archivo fotografico). Novedad provincial. 
Delforge (1999) ha descrito recientemente un taxón de floración tardía del agregado de Ophrys fusca Link. Más detalles sobre esta misma especie aparecen, casi a la vez, en Arnold (1999), quien mencionó su escasa presencia en el norte de la provincia de Alicante; aunque sin indicar localidad concreta. La revisión de citas previas y de material de $\mathrm{ABH}$ ha demostrado que esta especie está presente en la Sierra de Bèrnia y en la Sierra de l'Aixortà. Ophrys arnoldii se presenta en herbazales y claros de matorral, desde los 300 a los 1000 m de altitud, a menudo simpátrica con $O$. lupercalis J. DevillersTerschuren \& P. Devillers (- O. fuca auct.), pero muy fácil de distinguir por su floración mucho más tardía (entre mediados de abril y finales de mayo), por su mayor robustez y sus características florales.

\section{Ophrys bilunulata Risso}

ALICANTE: Muro de Alcoi, SE Port d'Albaida, 30SYH2098, $600 \mathrm{~m}$, 16-III-2000, M. R. Lowe (archivo fotográfico). Xalò, Xalò a Bèrnia K8.1, 30SYH5687, 520 m, 15-IV-2000, J. Piera (ABH 43878). Parcent, Coll de Raptes, 30S YH5590, 660 m, 05-III-2000, M. R. Lowe, J. Piera, M. B. Crespo, J. E. Arnold, J. Benito Ayuso \& C. E. Hermosilla (archivo fotográfico). Ibídem, 25-III2000, J. Piera (ABH 43128, ABH 43156). L'Orxa, SE L'Orxa, 30SYJ3402 $430 \mathrm{~m}$, 16-III-2000, M. R. Lowe (archivo fotográfico). Vall de Gallinera, Benirrama, camí Benimarsot, 30SYJ4402, $400 \mathrm{~m} 5$ IV-2000, J. Piera (ABH 43163, ABH 43164). Gata de Gorgos, carretera de Gata a Llíber, km 3.9, 31SBC4394, 140 m, 1-IV-2000, J. Piera (ABH 43158). $2^{\text {a }}$ citas provinciales y ampliación de área.

Este taxón fue indicado por primera vez para la provincia de Alicante por Delforge (1999), a partir de materiales provenientes de la Sierra del Montgó. No obstante, las referencias a $O$. funerea Viv. publicadas previamente por Lowe (1995) corresponden realmente a $O$. bilunulata. Nuestras observaciones sobre el terreno demuestran que se trata de un planta poco frecuente, distribuida principalmente por las áreas diánicas de la provincia.

\section{Serapias parviflora Parl.}

ALICANTE: Vall d'Alcalà, El Teular, carretera CV-712, km 6.9, 30SYH3998, 605 m, 31 V-2000, M.R. Lowe (ABH 43877). $2^{\text {a }}$ cita provincial y ampliación de área.

Planta conocida únicamente de los alrededores de Jávea-Xàbia (31SBC59), de donde ha sido indicada por diversos autores (cf. Piera \& Crespo, 1999). Con esta nueva localidad se amplía hacia las comarcas interiores el área de distribución de esta rara planta de la flora valenciana. Sería interesante establecer una microrreserva de flora (cf. Laguna, 1996) para segurar la conservación de esta especie, que se encuentra seriamente amenazada en Alicante (Piera \& Crespo, 1999).

\section{BIBLIOGRAFÍA}

ARNOLD, J. E. -1999- La problématique des groupes d'Ophrys fusca et d'Ophrys omegaifera en Catalogne et dans le Pays Valencien (Espagne). Natural. belges 80 (orchid 12): 120 140, 275.

BENITO AYUSO, J. y C. E. HERMOSILLA - 1998Dos nuevas especies ibéricas, Epipactis cardina y Epipactis hispanica, más alguno de sus híbridos: Epipactis $x$ conquensis (E. cardina $\times$ E. parviflora), y Epipactis $\times$ populetorum $(E$. helleborine $\times$ E. hispanica). Est. Mus. Cienc. Nat. Alava: 13: 103-115.

DELFORGE, P. -1994-Guide des Orchidées d'Europe d'Afrique du Nord et du ProcheOrient. Delachaux et Niestlé. Lausanne et Paris.

DELFORGE, P. - 1999- Ophrys arnoldii et Ophrys lucentina, deux espèces nouvelles du groupe d'Ophrys fusca. Natural. belges 80 (orchid 12): 244-260, 277-278.

LAGUNA, E. -1996-Conservación in situ mediante microrreservas de flora en la Comunidad Valenciana. Bol. Real Soc. Esp. Hist. Nat. Tomo Extraord. 125 Aniversario: 379-381. Madrid.

LOWE, M. R. -1995- Les Orchidées de la province d'Alicante, Espagne. Natural. belges 76 (orchid 8): 78-83.

MATEO, G. y CRESPO, M.B. -1998-Manual para la determinación de la flora valenciana. Monogr. Fl. Montiber. 3. Valencia.

PIERA, J. y M.B. CRESPO -1999- Cuatro plantas raras de la flora valenciana, muy seriamente amenazadas. Fl. Montiber. 13: 50-52.

RIGUAL, A. -1972- Flora y vegetación de la provincia de Alicante. Inst. Estud. Alicantinos. Alicante.

SERRA, L. y G. MATEO - 1994-Sobre la presència de Cephalanthera damasonium (Miller) Druce 
al País Valencià. Butll. Inst. Cat. Hist. Nat.. 61: 81-82.

SERRA, L., C. FABREGAT, J. JUÁREZ, P. PÉREZ ROVIRA, V. DELTORO, J. PÉREZ BOTELLA, A. OLIVARES, B. PÉREZ ROCHER, M. C. ESCRIBÁ y J. BENITO AYUSO -2000Adiciones y correcciones a la orquidoflora Valenciana, I. Flora Montiberica 15: 10-20.

SOLANAS, J. L. -1998- Flora, vegetació $i$ fitogeografia de la Marina Baixa. Servicio de Publicaciones de la Universidad de Alicante. Alicante.
Aceptado para su publicación en julio de 2000

Dirección de los autores: M.B. CRESPO: Centro Iberoamericano de la Biodiversidad (CIBIO), Universidad de Alicante. Apartado 99. E-03080 Alicante. Correo electrónico: crespo@carn.ua.es. J. PIERA: I.E.S. ${ }^{\circ} 1$ "Bellaguarda". Partida Garganes s/n. E-03590 Altea (Alicante). Correo electrónico: atjopiol@altea.infoville.net. M.R. LOWE: School House, 17. The Village, Brancepeth, Durham DH7 8DG, United Kingdom. Correo electrónico: MichaelRLowe@orchid.freeserve.co.uk.

\title{
89. ADICIONES A LA FLORA DE MURCIA. II
}

\author{
Pedro SÁNCHEZ GÓMEZ, Antonio Félix CARRILLO, Miguel Ángel CARRIÓN, \\ Antonio HERNÁNDEZ y Juan Francisco JIMÉNEZ
}

New records and corrections for the flora of Murcia.

Palabras clave. Flora vascular, corología, Murcia, España.

Key words. Vascular Flora, corology, Murcia, Spain.

Como consecuencia de las nuevas recolecciones realizadas en la provincia de Murcia, han sido localizados una serie de táxones que sirven para mejorar o ampliar los ya incluidos en Flora de Murcia (cf. Sánchez Gómez et al, 1998a),

Asperula cynanchica $\mathrm{L}$.

*MURCIA: Moratalla, Cortijo de la Umbría,
30SWH9424,1040 m, herbazal. M. A. Carrión, J. A. Jiménez, A. F. Carrillo \& P. Sánchez Gómez. 18VI-1998. MGC 48191. Elemento holártico.

Astragalus cavanillesii Podlech

*MURCIA: Moratalla, proximidades del arroyo de la Rogativa, 30SWH6826, $1100 \mathrm{~m}$, herbazal subnitrófilo. C. Selma. 6-VI-1981. MUB20306. Especie hasta el momento tan sólo conocida de La Sagra (Granada). Este taxon ha sido

Trabajo financiado con cargo a los proyectos FLP/9/FS97 de la Fundación SÉNECA y PB97-1061 de la DGES. 